Luisa Agnello, Alessandro lacona, Bruna Lo Sasso, Concetta Scazzone, Michele Pantuso, Rosaria Vincenza Giglio, Caterina Maria Gambino, Anna Maria Ciaccio, Giulia Bivona, Matteo Vidali and Marcello Ciaccio*

\title{
A new tool for sepsis screening in the Emergency Department
}

https://doi.org/10.1515/cclm-2021-0208

Received February 16, 2021; accepted March 30, 2021;

published online April 13, 2021

\section{Abstract}

Objectives: In this study, we developed and evaluated the diagnostic accuracy of the Sepsis Index for early sepsis screening in the Emergency Department (ED).

Methods: Sepsis Index is based on the combination of monocyte distribution width (MDW) and mean monocyte volume (MMV). Sepsis Index $\geq 1$ was selected to define sepsis. We tested its diagnostic accuracy in an ED population stratified in four groups: controls, Systemic Inflammatory Response Syndrome (SIRS), infection, and sepsis, according to Sepsis-2 criteria.

Results: Patients with sepsis displayed higher median Sepsis Index value than patients without sepsis. At the receiver operating characterictis (ROC) curve analysis for the prediction of sepsis, the area under the curve (AUC) of MDW and Sepsis Index were similar: 0.966 (95\%CI

Luisa Agnello and Alessandro lacona contributed equally to this work.

*Corresponding author: Prof. Marcello Ciaccio, Department of Biomedicine, Neurosciences and Advanced Diagnostics, Institute of Clinical Biochemistry, Clinical Molecular Medicine and Laboratory Medicine, University of Palermo, Palermo, Italy; and Department of Laboratory Medicine, University Hospital “P. Giaccone”, Palermo, Italy, E-mail: marcello.ciaccio@unipa.it

Luisa Agnello, Concetta Scazzone, Michele Pantuso, Rosaria Vincenza Giglio, Caterina Maria Gambino and Giulia Bivona, Department of Biomedicine, Neurosciences and Advanced Diagnostics, Institute of Clinical Biochemistry, Clinical Molecular Medicine and Laboratory Medicine, University of Palermo, Palermo, Italy Alessandro lacona, Department of Laboratory Medicine, University Hospital "P. Giaccone”, Palermo, Italy

Bruna Lo Sasso, Department of Biomedicine, Neurosciences and Advanced Diagnostics, Institute of Clinical Biochemistry, Clinical Molecular Medicine and Laboratory Medicine, University of Palermo, Palermo, Italy; and Department of Laboratory Medicine, University Hospital "P. Giaccone", Palermo, Italy

Anna Maria Ciaccio, University Hospital “P. Giaccone”, Palermo, Italy Matteo Vidali, Foundation IRCCS Ca’ Granda Ospedale Maggiore Policlinico, Milan, Italy
0.947-0.984), and 0.964 (95\%CI 0.942-0.985), respectively. Sepsis Index showed increased specificity than MDW (94.7 vs. $90.6 \%$ ), without any decrease in sensitivity (92.0\%). Additionally, LR+ increased from 9.8 (MDW) to 17.4 (Sepsis Index), without any substantial change in LR- (respectively 0.09 vs. 0.08). Finally, PPV increased from 0.286 (MDW) to 0.420 (Sepsis Index).

Conclusions: Sepsis Index improves the diagnostic accuracy of MDW alone for sepsis screening.

Keywords: biomarker; Emergency Department; mean monocyte volume (MMV); monocyte distribution width (MDW); sepsis.

\section{Introduction}

Sepsis represents a significant global health problem associated with a high rate of mortality [1-2]. It is defined as a life-threatening organ dysfunction due to a deregulated host response to infection. Accordingly, the rapid detection of infection is crucial in order to prevent the development of sepsis. However, this is challenging and the sepsis recognition is often delayed. Thus, useful biomarkers for identifying patients at risk of developing sepsis are strongly sought after. Among all molecules investigated [3-6], the role of monocyte distribution width (MDW) has recently emerged [7-13].

MDW detects the volumetric changes of monocytes, which represent an early phenomenon during sepsis. It has the great advantage of being easily measured as part of the complete blood count (CBC). In previous studies, we evaluated the diagnostic performance of MDW in the Emergency Department (ED) [7] and in the intensive care unit (ICU) [8]. Additionally, we established the MDW reference interval in a population of healthy blood donors [14]. Although MDW showed high sensitivity and specificity for sepsis screening in our studies, we noted, especially in the ED, that some individuals classified according to clinical data as control, Systemic Inflammatory Response Syndrome (SIRS), and infection subgroups showed MDW values ranging from the cut-off value of 
23-26. Thus, they were not correctly classified by MDW, resulting in false-positive. Therefore, we evaluated if other CBC parameters would better correctly classify patients in different subgroups. We found that patients with the same MDW, but belonging to different subgroups, showed different mean monocyte volume (MMV), a research parameter cell population data. However, since MMV alone has overall low diagnostic accuracy for sepsis detection [10], we developed an index, namely Sepsis Index, by combining MDW and MMV. Considering that MDW and MMV are both included in a routine $\mathrm{CBC}$, the estimation of the Sepsis Index could represent a rapid tool for supporting the early detection of sepsis in the acute setting, such as ED.

The aim of the present study was to assess the diagnostic performance of the Sepsis Index for sepsis screening in the ED.

\section{Materials and methods}

\section{Study population}

In this retrospective study, we further analysed data from a previous study on adult patients ( $\geq 18$ years of age) presenting to the ED of the University Hospital "P. Giaccone" of Palermo, Italy, from September to November 2019, whose initial evaluation included a CBC [7].

According to Sepsis-2 criteria [15], patients were classified into four groups: controls (patients without infection, SIRS and sepsis); SIRS (patients with at least two SIRS criteria); infection (patients without sepsis and with zero or one SIRS criterion); sepsis (patients with confirmed or suspected infection and SIRS). SIRS criteria were tachycardia, defined as heart rate $>90$ beats/min; tachypnea, defined as respiratory rate $>20$ breaths/min; fever or hypothermia, defined as temperature $>38$ or $<36^{\circ} \mathrm{C}$, respectively; and leukocytosis and leukopenia, defined as white blood cells $(\mathrm{WBC})>12 \times 10^{9} / \mathrm{L}$ and $<4 \times 10^{9} / \mathrm{L}$, respectively. The infection was defined according to clinical, imaging, and laboratory tests findings.

\section{Haematological analysis}

After the laboratory analysis ordered for the clinical purpose was performed, all whole-blood samples collected in $\mathrm{K}_{3}$-EDTA were analysed on the UniCel DxH 900 haematology analyzer (Beckman Coulter, Inc., Brea, California) within $2 \mathrm{~h}$ from the collection, as recommended by the manufacturer. The software version of the DxH 900 was 1.1.0. This instrument has a unique capability to measure specific cell volume parameters and the distribution of cell volumes within a group of cells, as previously reported [7].

\section{Sepsis index}

As stated in the introduction, we observed that patients with MDW values ranging 23-26 were not correctly classified according to clinical subgroups (controls, SIRS, and infection). The increase of MDW is associated with the appearance of a circulating monocytes population characterised by increased volume [16]. Based on such evidence, we evaluated the complex relationship between MDW and MMV (Figure 1). The graph clearly shows the positive, although characterized by high dispersion, association between MDW and MMV. When stratifying patients according to the presence of sepsis (septic vs. non-septic), MDW and MMV alone identified two clusters (septic vs. non-septic), with an overlapping area. Interestingly, when MDW is plotted against MMV for all observations, the area of overlapping was significantly reduced. In order to increase the specificity of MDW, we developed a polynomial function that could interpolate all points of the cluster of septic patients who had the following characteristics: i) a MDW value $\geq 23$; ii) the lowest MDW value with the same MMV. When applying the polynomial function, a theoretical MDW (MDWth) value was obtained for each MMV value. Sepsis Index is calculated as the MDW/MDWth ratio (Figure 2) and a value $\geq 1$ was selected to define sepsis.

\section{Statistical analysis}

Statistical analyses were performed by SPSS statistical software v.17.0 (SPSS Inc., Chicago, IL, USA) and R Language v.4.0.3 (R Foundation for Statistical Computing, Vienna, Austria). Normality distribution was assessed preliminarily by q-q plot, Kolmogorov-Smirnov and
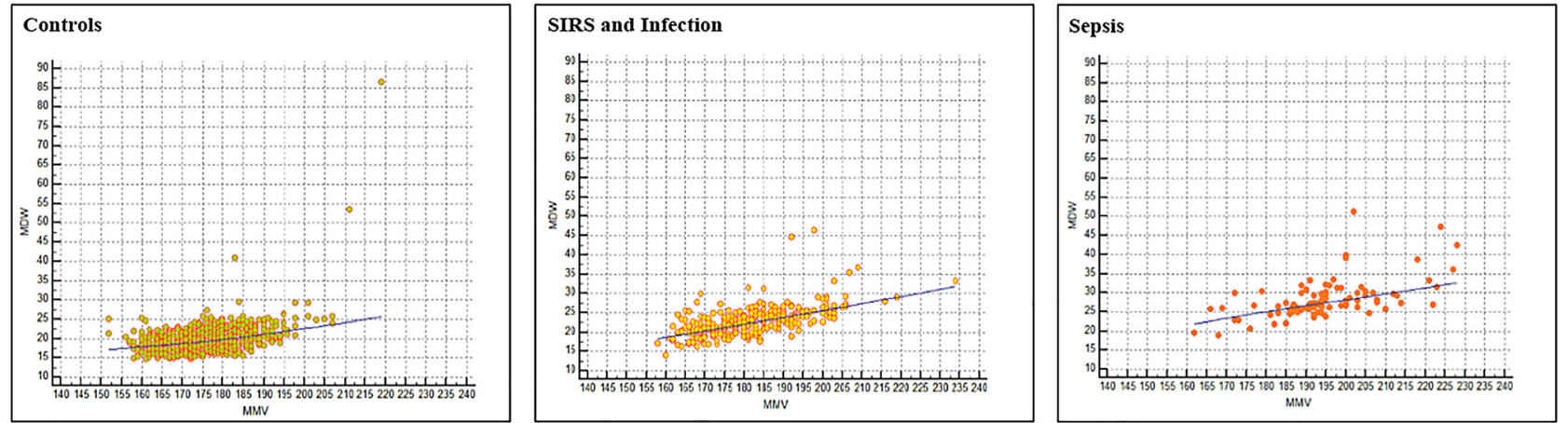

Figure 1: Dispersion plot of MDW and MMV in the different subgroups.

Correlation coefficients: controls, rho 0.345, p<0.001; SIRS and infection, rho 0.583, p<0.001; sepsis, rho 0.554, $p<0.001$. 


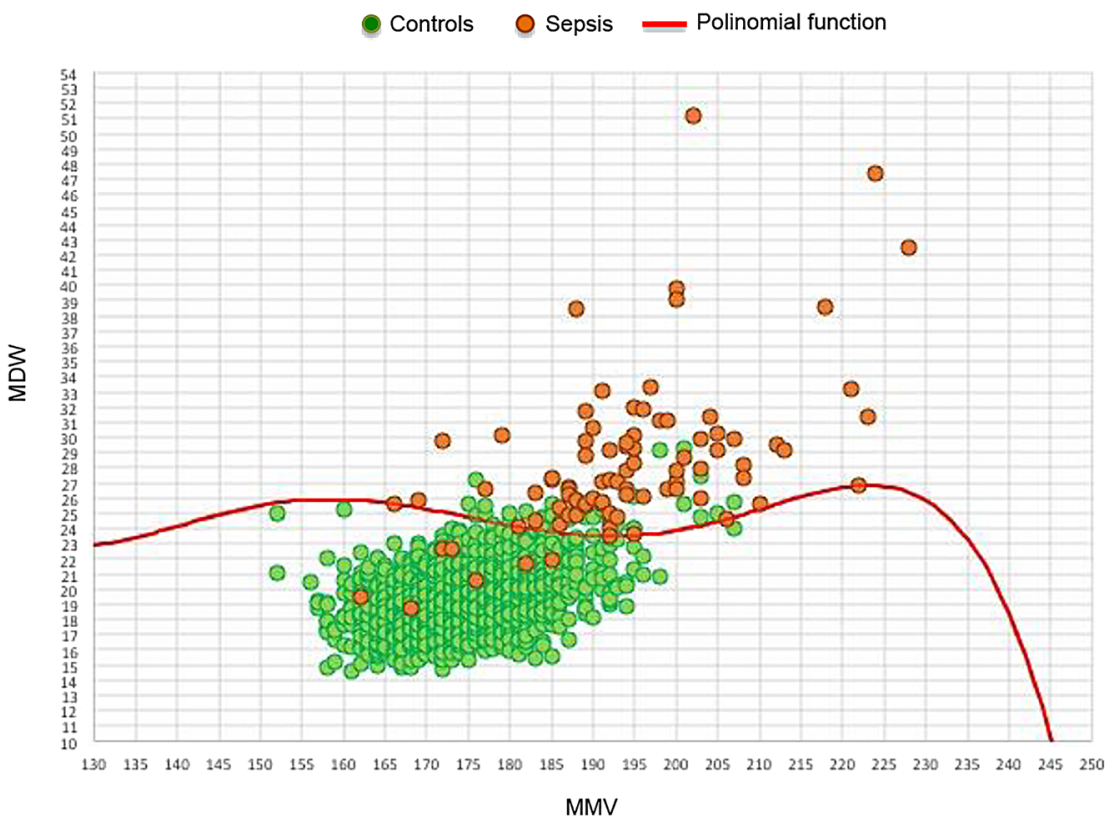

Figure 2: Dispersion plot of MDW and MMV in controls and septic patients.

Orange dots are septic patients, green dots are controls, red line is the interpolation line.
Shapiro-Wilk tests. Quantitative variables were expressed as the median and interquartile range (IQR). Differences between groups for continuous and categorical variables were estimated respectively by nonparametric Kruskal-Wallis test (if $>2$ groups) or Mann-Whitney U-test (with Bonferroni's correction when needed). Diagnostic accuracy for the prediction of sepsis was evaluated by receiver operating characteristics (ROC) curve analysis and reported as area under the curve (AUC) and 95\% confidence interval. Differences between the AUCs were evaluated by the DeLong method.

\section{Results}

Two thousand two hundred fifteen patients (controls $n=1855$, SIRS $n=100$, infection $n=172$, sepsis $n=88$ ) were evaluated for haematological parameters, including white blood cells (WBC), neutrophils (NEU), mean neutrophil volume (MNV), standard deviation neutrophil volume (SDNV), monocytes (MO), MMV, standard deviation monocytes volume (SDMV), MDW, and Sepsis Index (Table 1). Controls displayed significantly lower levels of WBC, NEU, MO, MNV, SDNV, MMV, SDMV, MDW, and Sepsis Index than all other subgroups (all groups vs. controls $\mathrm{p}<0.001$ with Bonferroni's correction). Patients in the infection subgroup showed significant lower levels of WBC $(p<0.001)$ and NEU $(p<0.001)$ than patients with SIRS and significant lower levels of WBC $(p<0.001)$, NEU $(p<0.001)$, MNV ( $p=0.006)$, SDNV ( $p<0.001)$, and MMV $(p<0.001)$ than patients with Sepsis. SIRS and Sepsis were instead significantly different for MNV ( $p<0.001)$, SDNV ( $p<0.001), M M V$ $(\mathrm{p}<0.001)$, and SDMV $(\mathrm{p}<0.001)$ (Table 1$)$.

MDW values were significantly different among groups $(\mathrm{p}<0.001)$; all comparisons, taking into account

Table 1: Hematological and biochemical data in the four subgroups defined according to Sepsis-2 criteria.

\begin{tabular}{|c|c|c|c|c|c|}
\hline Parameter, median, IQR & Controls $\mathrm{n}=\mathbf{1 8 5 5}$ & SIRS $n=100$ & Infection $n=172$ & Sepsis $n=88$ & p-Value \\
\hline WBC, $\times 10^{9} / \mathrm{L}$ & $9.00(7.40-11.10)$ & $14.15(12.65-16.58)$ & $10.10(7.60-13.05)$ & $13.35(10.60-19.25)$ & $<0.001$ \\
\hline $\mathrm{NEU}, \times 10^{9} / \mathrm{L}$ & $5.80(4.50-7.70)$ & $11.10(8.90-13.38)$ & $6.95(4.90-9.90)$ & $10.30(8.60-16.00)$ & $<0.001$ \\
\hline $\mathrm{MO}, \times 10^{9} / \mathrm{L}$ & $0.70(0.50-0.90)$ & $1.00(0.70-1.30)$ & $0.85(0.60-1.10)$ & $0.90(0.60-1.38)$ & $<0.001$ \\
\hline MNV & $149(145-154)$ & $152(147-157)$ & $154(148-159)$ & $157(152-165)$ & $<0.001$ \\
\hline SDNV & $18.29(17.48-19.24)$ & $19.47(18.31-20.60)$ & $19.29(18.35-20.49)$ & $21.63(19.49-23.41)$ & $<0.001$ \\
\hline MMV & $174(170-179)$ & $178(172-185)$ & $183(173-191)$ & $193(187-200)$ & $<0.001$ \\
\hline SDMV & $19.25(18.05-20.61)$ & $21.14(19.36-22.91)$ & $22.54(21.09-24.37)$ & $25.36(24.24-26.92)$ & $<0.001$ \\
\hline MDW & $19.0(17.8-20.4)$ & $21.3(19.5-23.3)$ & $23.2(21.0-25.0)$ & $27.0(25.6-29.8)$ & $<0.001$ \\
\hline Sepsis Index & $0.77(0.71-0.83)$ & $0.87(0.79-0.97)$ & $0.96(0.86-1.04)$ & $1.13(1.06-1.24)$ & $<0.001$ \\
\hline
\end{tabular}

IQR, interquartile range; SIRS, Systemic Inflammatory Response Syndrome; WBC, white blood count; NEU, neutrophils; MNV, mean neutrophil volume; SDNV, standard deviation neutrophil volume; MO, monocytes; MMV, mean monocyte volume; SDMV, standard deviation monocyte volume; MDW, monocyte distribution width. Statistical significance was calculated according to nonparametric Kruskal-Wallis test. 
Bonferroni's correction, were also significant (all $\mathrm{p}<0.001$ ). The Sepsis subgroup displayed the highest median MDW levels of 27.0 (25.6-29.8).

Using the upper limit of the reference interval of MDW previously defined in a population of healthy blood donors (23.0) [13], we found out that in the ED population, sensitivity and specificity for sepsis were respectively $92.0 \%(81 / 88)$ and $90.6 \%(1926 / 2127)$.

As with MDW, patients with sepsis also displayed higher median Sepsis Index value than patients without sepsis, including controls, SIRS and infection subgroups [controls: 0.77 (0.71-0.83); SIRS: 0.87 (0.79-0.97); infection: 0.96 (0.86-1.04); sepsis: $1.13(1.06-1.24), \mathrm{p}<0.001]$.

At the ROC curve analysis for the prediction of sepsis, MDW and Sepsis Index showed the best AUC (Table 2). Figure 3 shows the comparison of the AUC of MMV, MDW and Sepsis Index. Additionally, MDW and Sepsis Index showed likelihood ratio (LR)+, LR-, positive predictive value (PPV) and negative predictive value (NPV) of 9.7, $0.09,0.286,0.996$ and 17.4, 0.08, 0.420, 0.997, respectively.

According to DeLong's test and taking into account Bonferroni's correction, the AUCs of MDW ( $<<0.001)$ and Sepsis Index $(\mathrm{p}<0.001)$ were significantly higher than the AUC of MMV; however, no significant difference was instead observed between the AUCs of MDW and Sepsis Index.

Although the AUC of Sepsis Index was not significantly different from the AUC of MDW, the specificity of Sepsis Index using a cut-off of 1 was higher than MDW (94.7 vs. 90.6\%), without any decrease in sensitivity (92.0\%). Accordingly, LR+ increased from 9.7 (MDW) to 17.4 (Sepsis index), without any substantial change in LR- (respectively

Table 2: Area under the curve (AUC) of SDNV, SDMV, MDW, MMV, MNV, Sepsis Index for identifying patients at high risk of sepsis in the ED.

\begin{tabular}{lrr}
\hline CBC parameter & AUC & 95\% Cl \\
\hline WBC & 0.744 & $0.725-0.762$ \\
NEU & 0.777 & $0.759-0.794$ \\
MO & 0.624 & $0.603-0.644$ \\
MNV & 0.749 & $0.693-0.804$ \\
SDNV & 0.843 & $0.796-0.890$ \\
MMV & 0.892 & $0.847-0.936$ \\
SDMV & 0.956 & $0.938-0.974$ \\
MDW & 0.966 & $0.947-0.984$ \\
Sepsis Index & 0.964 & $0.942-0.985$ \\
\hline
\end{tabular}

WBC, white blood count; NEU, neutrophils; MO, monocytes; MNV, mean neutrophil volume; SDNV, standard deviation neutrophil volume; MMV, mean monocyte volume; SDMV, standard deviation monocyte volume; MDW, monocyte distribution width.

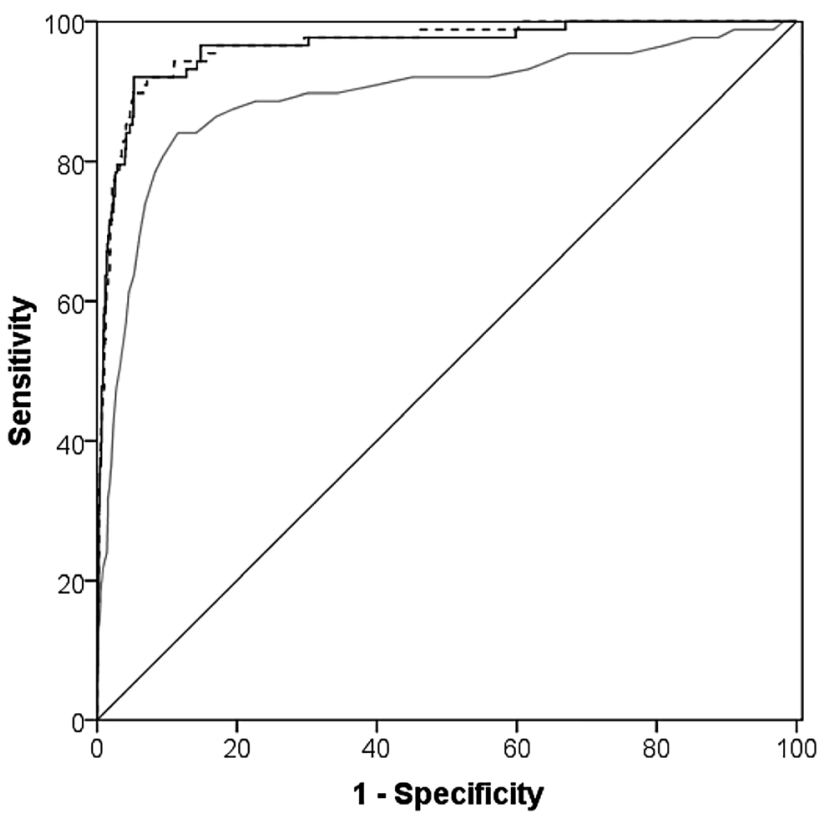

Figure 3: ROC curve analysis of MDW (black dashed), Sepsis Index (black solid) and MMV (solid grey) in sepsis prediction.

0.09 vs. 0.08). Specifically, in the MDW range $23-26$ among controls, infections and SIRS, 77/1855 (4.2\%), 61/172 (35.5\%) and 24/100 (24\%), respectively, resulted to be false positives. Applying the Sepsis Index $(\geq 1)$ to the incorrectly classified patients based on MDW ranging 23-26 we found that 50 out 77 controls, 24 out 61 with infection and 15 out 24 with SIRS were correctly reclassified as being without sepsis.

\section{Discussion}

In this large observational cohort study, we developed and evaluated the diagnostic accuracy of an index, namely Sepsis Index, for the early detection of patients at risk of sepsis in ED. Sepsis Index is based on the combination of two CBC parameters related to the morphological characteristics of monocytes, the MDW, a measure of the monocyte anisocytosis, and the MMV, a measure of the mean volume of monocytes. Since monocytes are the first line of response to infections, CBC parameters related to monocytes are promising candidates for early sepsis detection.

The main findings of our study can be summarized as follows: i) Sepsis Index gradually increased across control, SIRS, infection and sepsis groups; the latter had the highest value of Sepsis Index; ii) ROC curve analysis showed that Sepsis Index had an exceptional diagnostic accuracy for the prediction of sepsis; iii) Sepsis Index and MDW showed 
comparable AUC and sensitivity. However, when patients were stratified according to MDW and Sepsis Index best cut-offs identified by Youden Index, Sepsis Index displayed better diagnostic performance than MDW. In particular, Sepsis Index showed an increased specificity, PPV and LR+. Overall, Sepsis Index improves the diagnostic accuracy of MDW alone for the early identification of patients at high risk of sepsis in the ED. Indeed, Sepsis Index provides complete information on the monocytes morphological characteristics allowing better capturing the morphological variability of the monocyte population during the early stages of sepsis. Thus, Sepsis Index could represent a valuable tool for identifying patients at high risk of sepsis, whose diagnosis should be confirmed by clinical, radiological and laboratory assessment.

Noteworthy, we found that MDW has exceptional diagnostic performance in comparison to previous studies [10-11]. This could be explained by the characteristics of the study population, which present different clinical conditions. Specifically, the MDW cut-off value of 23 shows optimal diagnostic accuracy in a population with a low prevalence of infection and sepsis. In other clinical settings or in ED with a higher prevalence of such conditions, MDW could display different accuracy when using the cut-off value of 23.

Sepsis Index, in comparison with MDW, reduces the rate of false-positive, thus representing an appealing tool, which increases the specificity of the MDW without compromising the sensitivity. Noteworthy, Sepsis Index is based on the relationship between two CBC parameters, which are automatically measured. Therefore, it does not require additional cost and analysis. Overall, Sepsis Index represents a very promising tool for sepsis detection in the ED. If its diagnostic accuracy will be confirmed in larger and multicentre cohort studies, it could be automatically calculated by the haemocytometer and included as a parameter of the CBC. Thus, it could be easily available to all clinicians during the first evaluation of a patient.

Remarkable, Sepsis Index was established in blood samples drawn in $\mathrm{K}_{3}$-EDTA. Since the manufacturer reported the influence of the anticoagulant on MDW values, the Sepsis Index should be applied only on samples drawn in $\mathrm{K}_{3}$-EDTA tubes. Further studies are required to test its accuracy also for $\mathrm{K}_{2}$-EDTA drawn blood samples.

\section{Conclusions}

Sepsis Index could represent an innovative tool for easily and early identifying patients at risk of sepsis in the ED. If validated in further studies, Sepsis Index could be implemented in the $\mathrm{CBC}$ report.

Research funding: None declared.

Author contributions: All authors have accepted responsibility for the entire content of this manuscript and approved its submission.

Competing interests: Authors state no conflict of interest. Informed consent: Not applicable.

Ethical approval: The study was performed in accordance with the tenets of the Helsinki Declaration (as revised in 2013), and has been approved by the Ethics Committee of the University Hospital of Palermo (nr 07/2019).

\section{References}

1. Rudd KE, Johnson SC, Agesa KM, Shackelford KA, Tsoi D, Kievlan DR, et al. Global, regional, and national sepsis incidence and mortality, 1990-2017: analysis for the Global Burden of Disease Study. Lancet 2020;395:200-11.

2. Jordan Kempker A, Rudd KE, Wang HE, Martin GS. Sepsis epidemiology across the international classification of diseases, 9th edition, to international classification of diseases, 10th edition, chasm-A direct application of the institute for health metrics and evaluation case definition to hospital discharge data. Crit Care Med 2020;48:1881-4.

3. Giulia B, Luisa A, Concetta S, Bruna LS, Chiara B, Marcello C. Procalcitonin and community-acquired pneumonia (CAP) in children. Clin Chim Acta 2015;451:215-8.

4. Bellia C, Agnello L, Lo Sasso B, Bivona G, Raineri MS, Giarratano A, et al. Mid-regional pro-adrenomedullin predicts poor outcome in non-selected patients admitted to an intensive care unit. Clin Chem Lab Med 2019;57:549-55.

5. Agnello L, Bivona G, Parisi E, Lucido GD, lacona A, Ciaccio AM, et al. Presepsin and midregional proadrenomedullin in pediatric oncologic patients with febrile neutropenia. Lab Med 2020;51: 585-91.

6. Kim MH, Choi JH. An update on sepsis biomarkers. Infect Chemother 2020;52:1-18.

7. Agnello L, Bivona G, Vidali M, Scazzone C, Giglio RV, lacolino G, et al. Monocyte distribution width (MDW) as a screening tool for sepsis in the Emergency Department. Clin Chem Lab Med 2020; 58:1951-7.

8. Agnello L, Sasso BL, Giglio RV, Bivona G, Gambino CM, Cortegiani A, et al. Monocyte distribution width as a biomarker of sepsis in the intensive care unit: a pilot study. Ann Clin Biochem 2021;58:70-3.

9. Polilli E, Sozio F, Frattari A, Persichitti L, Sensi M, Posata R, et al. Comparison of monocyte distribution width (MDW) and procalcitonin for early recognition of sepsis. PloS One 2020;15: e0227300.

10. Crouser ED, Parrillo JE, Seymour C, Angus DC, Bicking K, Tejidor L, et al. Improved early detection of sepsis in the ED with a novel monocyte distribution width biomarker. Chest 2017;152:518-26.

11. Crouser ED, Parrillo JE, Martin GS, Huang DT, Hausfater P, Grigorov I, et al. Monocyte distribution width enhances early 
sepsis detection in the emergency department beyond SIRS and qSOFA. J Intensive Care 2020;8:33.

12. Martinez-Iribarren A, Tejedor $X$, Sala Sanjaume À, Leis A, Doladé Botias $M$, Morales-Indiano C. Performance evaluation of the new hematology analyzer UniCel DxH 900. Int J Lab Hematol 2021. https://doi.org/10.1111/ijlh.13448 [Epub ahead of print].

13. Piva E, Zuin J, Pelloso M, Tosato F, Fogar P, Plebani M. Monocyte distribution width (MDW) parameter as a sepsis indicator in intensive care units. Clin Chem Lab Med 2021;59:1307-14.
14. Agnello L, Lo Sasso B, Bivona G, Gambino CM, Giglio RV, Iacolino G, et al. Reference interval of monocyte distribution width (MDW) in healthy blood donors. Clin Chim Acta 2020;510:272-7.

15. Levy MM, Fink MP, Marshall JC, Abraham E, Angus D, Cook D, et al. 2001 SCCM/ESICM/ACCP/ATS/SIS international sepsis definitions conference. Crit Care Med 2003;31:1250-6.

16. Yang J, Qiao M, Li Y, Hu G, Song C, Xue L, et al. Expansion of a population of large monocytes (atypical monocytes) in peripheral blood of patients with acute exacerbations of chronic obstructive pulmonary diseases. Mediators Inflamm 2018;2018:9031452. 